\title{
Simulation options for the airport terminal People Mover AGV system with ExtendSim 8
}

\author{
Gabriel Fedorko ${ }^{1, *}$, Vieroslav Molnár ${ }^{2}$, and Patrik Ščavnický ${ }^{1}$ \\ ${ }^{1}$ TU Košice F BERG, Logistics Department, Park Komenského 14, 04001 Košice, Slovakia \\ ${ }^{2}$ TU Košice FVT, Production Department, Bayerova 1, 08001 Prešov, Slovakia
}

\begin{abstract}
The use of AGV vehicles is expanding to an ever-wider range of diverse areas. In addition to securing effective logistics processes in the industry, AGV systems have been deployed in quite non-traditional areas, for example in health or transportation of persons. Although AGV systems intended for passenger transport do not belong to AGV systems according to the definition of VGA standards, they can still be regarded as a regular member of this category of vehicles. In order to ensure their reliable operation, the same tools as for conventional AGV systems can be used. Such tools include a computer simulation method. The paper presents the model of People Mover AGV in order to analyse passenger transport between airport terminals.
\end{abstract}

\section{Introduction}

Automated people-mover vehicles are no novelty in the airport ground transport. They are currently used at many airport terminals [1]. Their function is to securely facilitate safe passenger transport [2]. However, the area of people mover systems is associated with a whole range of responsibilities and challenges, regardless of whether it is the initial stage of their acquisition [3] or the area of their operation and maintenance [4]. In all these cases, it is essential to work with a sufficiently large set of information in order to create a sufficient information base for decision making and predictions [5]. Computer simulation as one of the analytical tools is the most suitable for this purpose [6]. In the area of AGV systems, computer simulation represents a major tool for data acquisition and implementation of a wide range of analyzes. For the purpose of creating simulation models, a broad base of simulation tools can be used. They differ from each other by their functionality, complexity and variability of the information transmitted. Simulation tools are particularly suitable for creating discrete simulation models of AGV systems [7]. These tools also include the ExtendSim 8 simulation program.

*Corresponding author: gabriel.fedorko@tuke.sk 


\section{People Mover AGV system specification}

The People Mover transport system (Fig. 1) is an autonomous transport technology that operates in the areas requiring the transport of persons at regular intervals. They are most often deployed, for example, in various airport terminals.
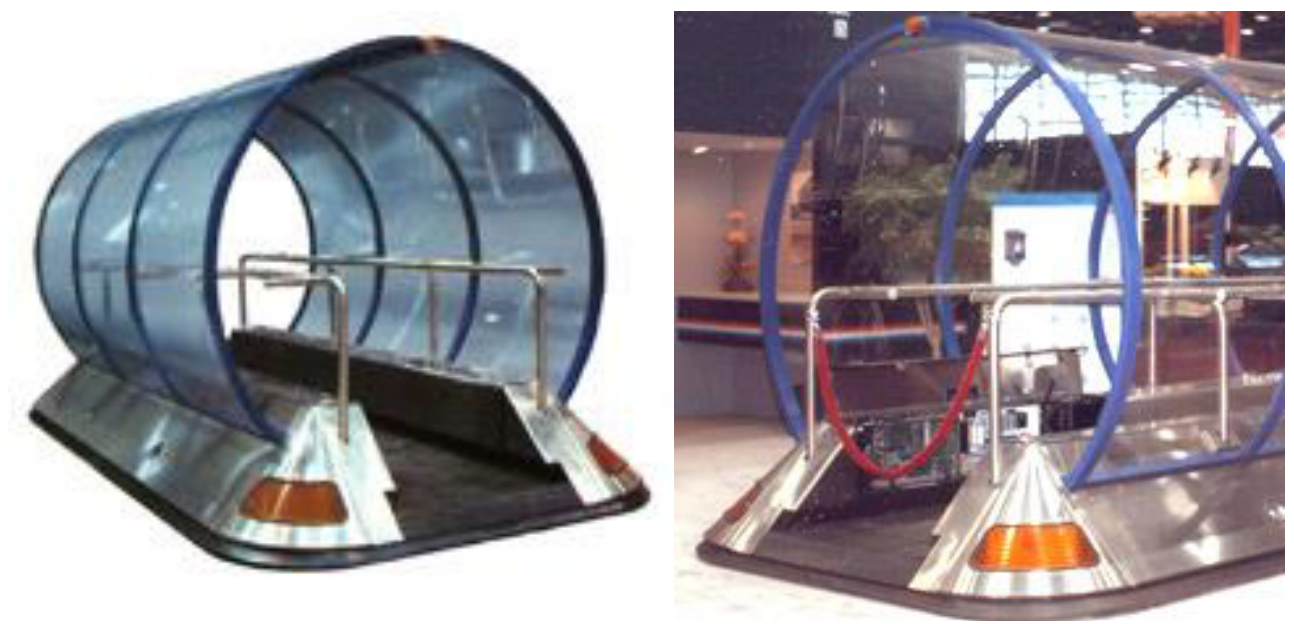

Fig. 1. Demonstration of People Mover transport system. Source: [8],[9]

Although it is an autonomous vehicle, its inclusion in the AGV systems group is questionable for many experts, because according to the VDA definition, it does not meet a basic condition, which is the transport of goods rather than persons. In spite of this uncertainty, this system is still included among AGV systems, as Ulrich points out [10].

\section{Creation of a simulation model}

The simulation of the AGV system puts high demands on the type of software tool used in terms of modeling and subsequent implementation of experiments. This is a very important factor that needs to be addressed. Many simulation software packages, which are practically applied in the field of discrete systems, are not suitable for the AGV area. This is due in particular to the different specificities that characterize AGV systems and which differentiate them from classical logistics processes. ExtendSim 8 is one of simulation programs suitable for AGV systems.

\subsection{Description of ExtendSim 8 program}

The ExtendSim 8 software simulation program is designed for computer simulation in a wide range of fields. It belongs to a group of object-oriented simulation programs that enable the creation of discrete, continuous and combined models.

ExtendSim 8 allows the creation of dynamic simulation models of various logistics processes. Within the created model, it provides a more detailed insight into more complicated processes. With the use of blocks and libraries it can create a simulation model with the option of further configuring individual parameters in blocks. It allows for the clear and rapid creation of simulation models with a high degree of unification and automation. 
The creation of simulation models in ExtendSim 8 is accomplished by block libraries where each block describes a particular part of the process or device. Using these blocks, we can build the scheme of the device or process that we need. In the simulation model, the blocks are connected via inputs and outputs. Individual blocks can be grouped together in order to create hierarchical structures that clarify and simplify the scheme.

\subsection{The People Mover AGV Simulation Model}

The simulation model was based on the following layout (Fig. 2), which shows the AGV route within a defined area of the airport terminal.

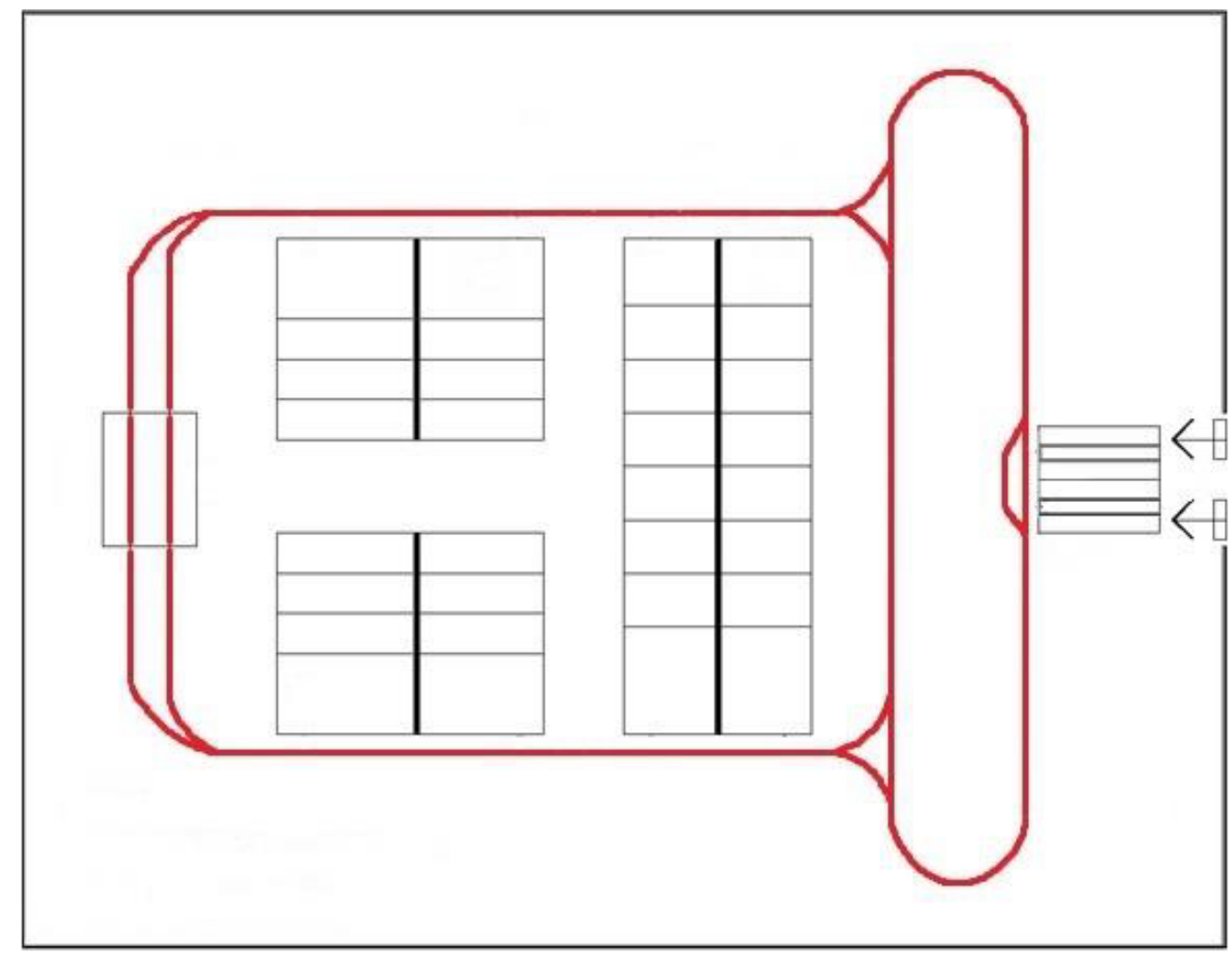

Fig. 2. Schematic representation of the People Mover AGV route.

Based on the analysis of the AGV system operation, a block diagram of the whole process was designed (Fig. 3).

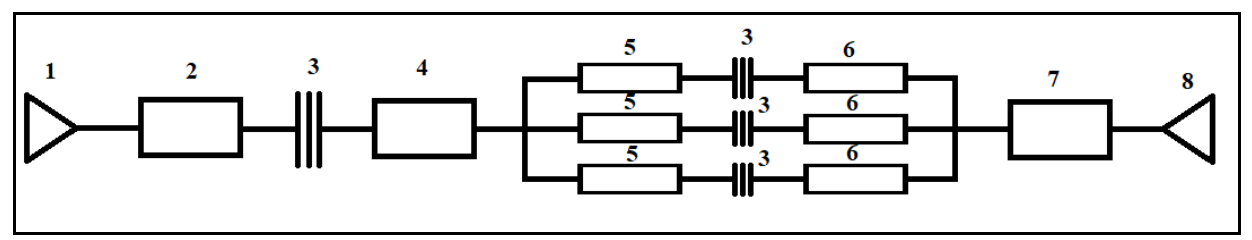

Fig. 3. Block diagram of distribution warehouse.

Within this block diagram, 8 functional components of the simulation model were considered:

1. "Start" block - to generate new requirements, in a particular scheme it generates an entity that represents AGV. 
2. "Delay" Block - the time of delay of the vehicle on the guiding line when moving to pick up passengers.

3. "Queue" Block - serves in a particular case as a parking space on which the vehicle waits until it is assigned a traffic task or some other action.

4. "Set Attribute" Block - a block that assigns an attribute to individual requirements; in this model, it assigns the vehicle its destination after the boarding of passengers, i.e., where the passengers will be transported to meet their transport requirements.

5. "Get Attribute" Block - the block that reads the attribute that was assigned to the requirement in the "Set Attribute" block.

6. "Delay2" Block - the time delay of the vehicle, from the point of picking up passengers to the place assigned to the vehicle as the boarding point for passengers.

7. "Savehouse" Block - the time delay of the vehicle, from the point of exit to the point which serves as a safe area for vehicles that have not been assigned or have already fulfilled their requirements.

8. "Exit" Block - the end block where the entity (AGV vehicle) that was created in the "Start" block is extinguished.

\subsection{Simulation model operation}

The People Mover AGV simulation begins with creating an entity in simulation time 0 and ends at 6000 minutes, i.e., 10 hours as total daily operating time. At time 0 , the AGV is located in the charging station, marked "N" in the diagram (Fig. 2). After running the simulation, the AGV begins to move to the place of boarding, where it will subsequently be assigned an exit point depending on the transport requirements. After the delay in driving the AGV vehicle, the passengers' exit is performed.

Passengers' exit in this simulation model can have three variants; exit at point A, exit at point $\mathrm{B}$ or exit at point $\mathrm{C}$.

When the AGV performs the exit of the passengers at the appropriate point, then it moves to the next boarding point.

Depending on the passengers' exit point, different time values are set in the simulation to move to the boarding point.

After this AGV removal, a further boarding of the passengers will take place and the AGV will take another run to the designated exit point. This continuous process in simulation continues until the simulation time reaches 6000 minutes, so the AGV operating time does not end at this point of time.

In the simulation model, individual blocks are grouped by hierarchical blocks that simplify the view of the entire simulation model. In the first hierarchical block (Fig. 4), a "Create" block is located creating an entity and an output connector that enables the entity to leave this hierarchical block.

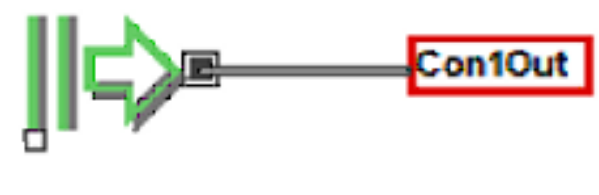

Fig. 4. Hierarchical "Start" Block.

Another hierarchical block (Fig. 5) represents passengers boarding and the assignment of the passengers' exit point. The hierarchical block also includes two input connectors and three output connectors. One of the input connectors serves as a connection for the output 
connector of the hierarchical "start" block, and the other input connector is connected to the hierarchical "new task" block. Output connectors serve as connections for hierarchical blocks corresponding to individual passenger exit points.

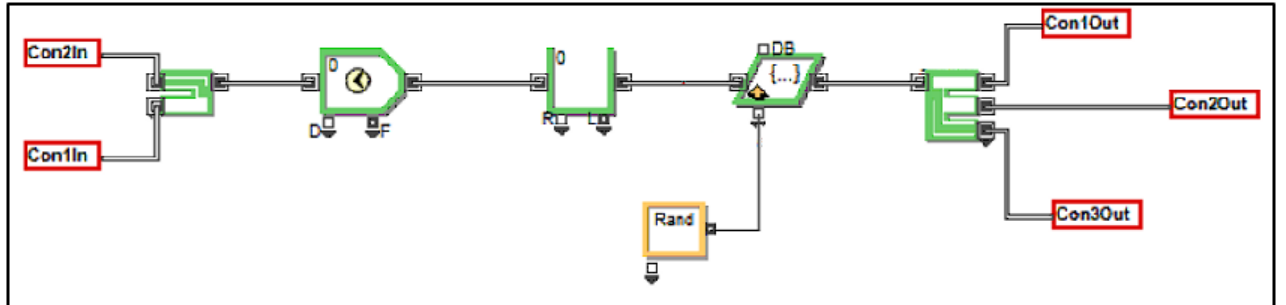

Fig. 5. Hierarchical "Loading" block.

The Hierarchical "Exit A" block (Fig. 6) contains blocks that define the weight of the goods and the time duration of the goods transport from loading to unloading in point $\mathrm{A}$. The input connector is a connection between the output connector of the "Boarding" hierarchical block and the output connector that follows the hierarchical "New Assignment" block. Also, the value of the AGV transport time for new passengers is addressed here. This time value depends on the exit point and is addressed in all hierarchical blocks dealing with the passengers' exit.

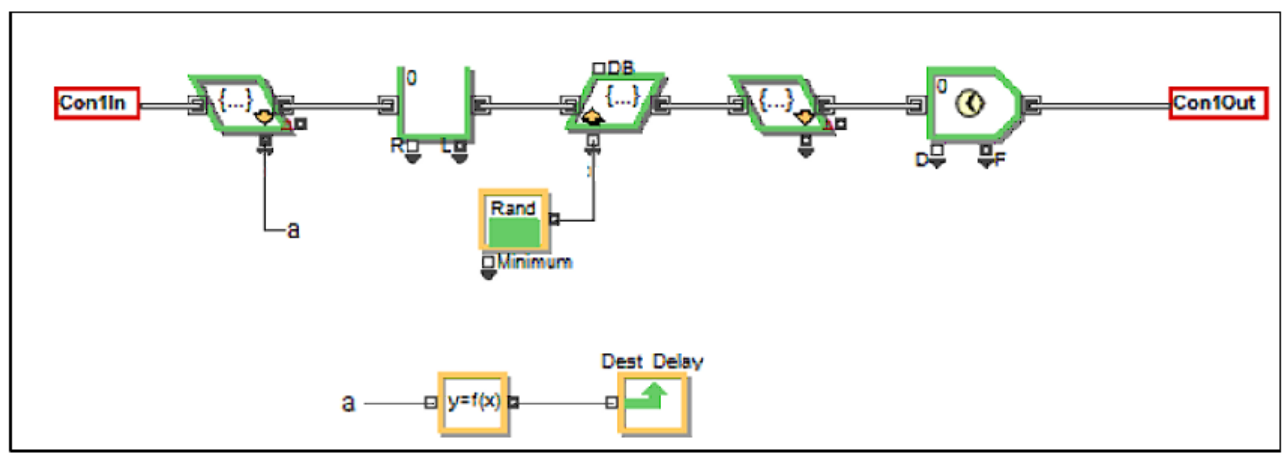

Fig. 6. Hierarchical „Exit A“block.

Similarly, the hierarchical "Exit B" and "Exit C" blocks are also created. The difference lies in time period in which the AGV moves to the exit point.

The last hierarchical block (Fig. 7) is the hierarchical "New assignment" block. It contains three input connectors that are connected to output connectors from each output point, and an output connector that connects this hierarchical block with the hierarchical "Boarding" block. It also contains a time delay, the value of which was set in the previous hierarchical blocks, depending on where the AGV was actually performing the exit of the passengers. 


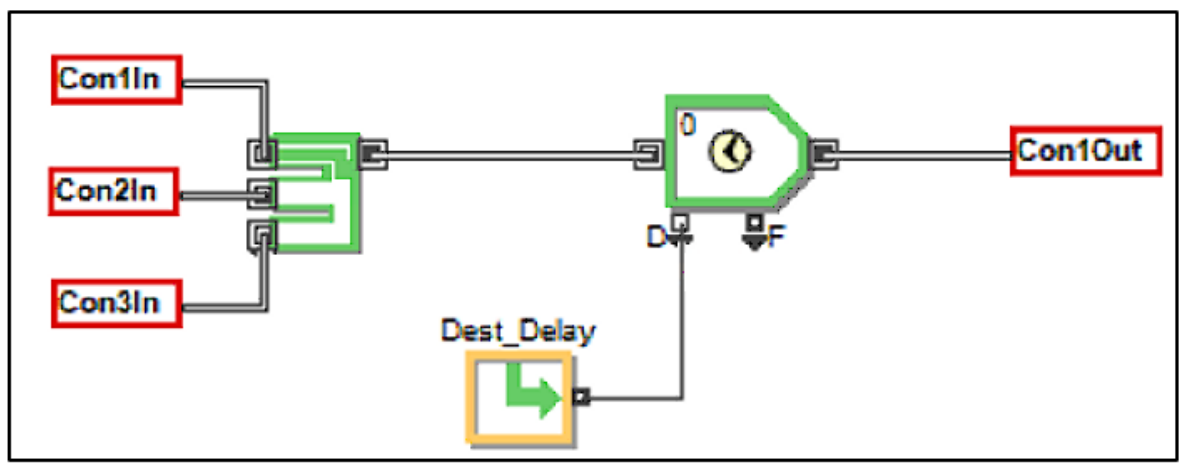

Fig. 7. Hierarchical "New Assignment" block.

\section{Conclusion}

The simulation model provides two basic graphical outputs. The first graphical output (Fig. 8) shows the figures for the individual numbers of passengers transported by the AGV system during the simulation. Passenger numbers are recorded separately for each exit point.

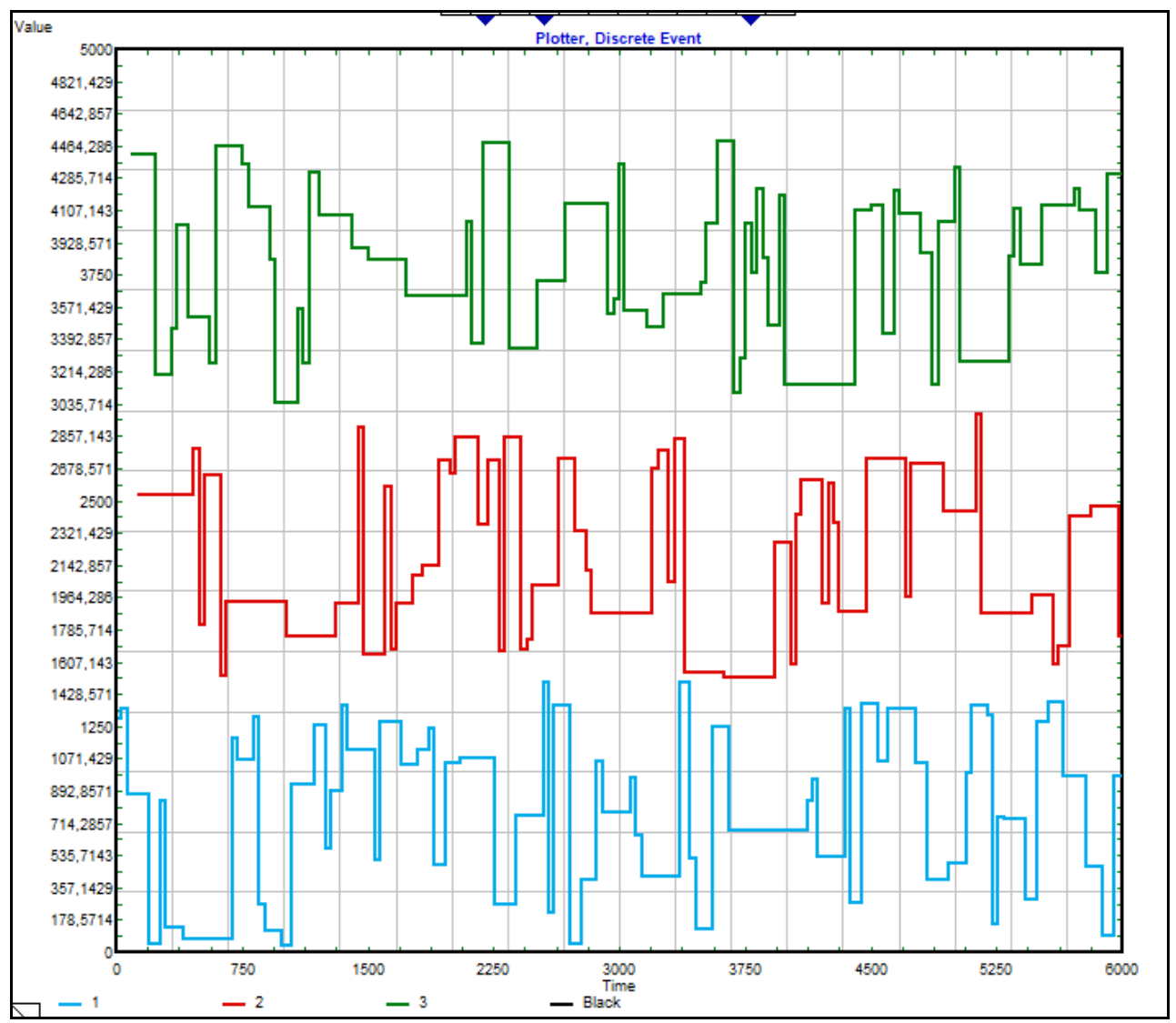

Fig. 8. Graphic output - Numbers of passengers transported. 
The second graphic output (Fig. 9) shows the AGV operating cost graph, the calculation of which depends on the distance traveled with the passengers and the number of passengers. This graph is also recorded separately for each exit point.

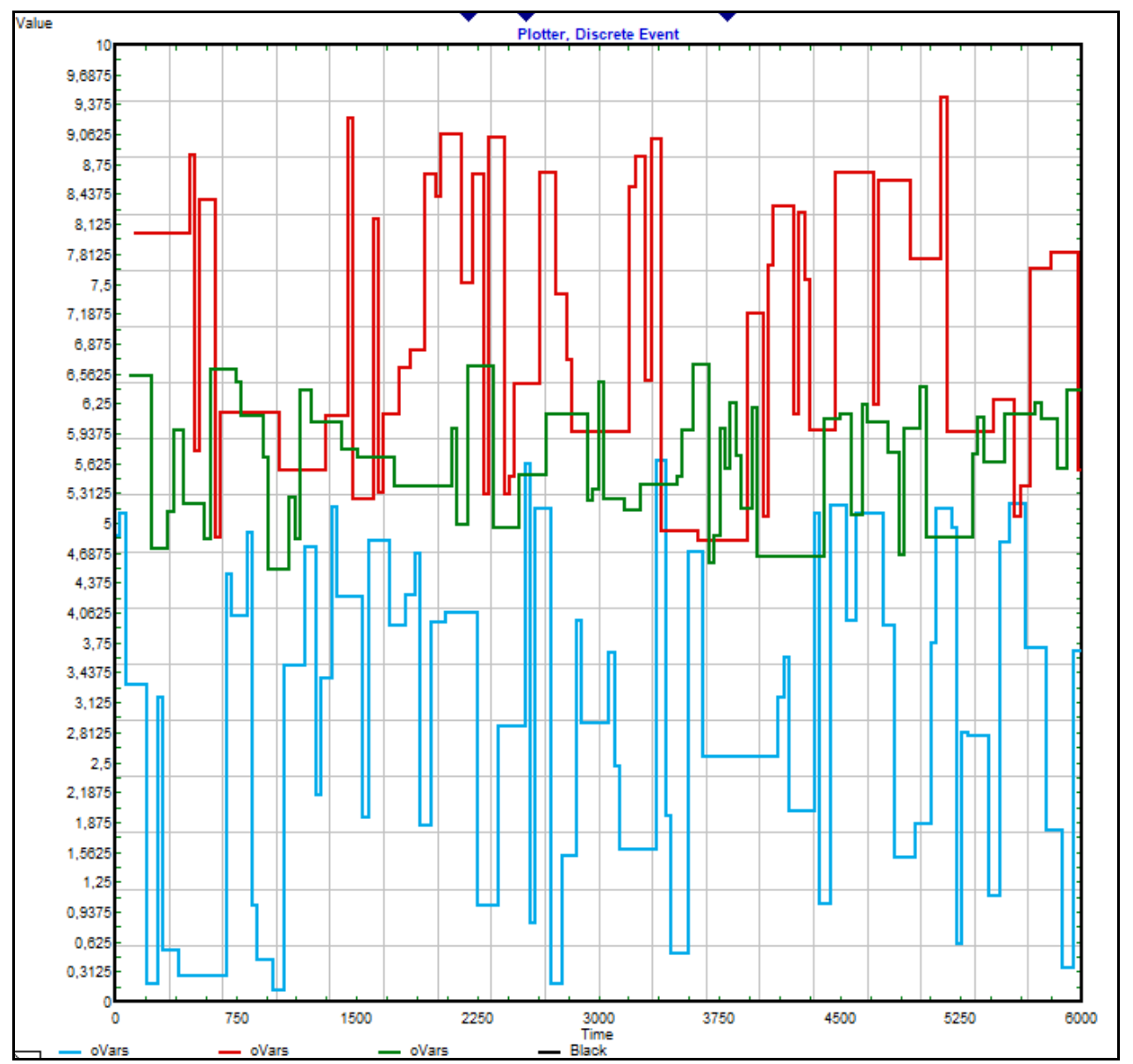

Fig. 9. Graphic Output - Operating Costs.

This simulation model primarily simulates the continuous work of one AGV during the operating time. It can serve as a suitable basis for creating additional simulation models and demonstrates the suitability of the ExtendSim 8 simulation program for modeling and simulating AGV systems.

The present paper is a part of research grant projects VEGA 1/0063/16, VEGA 1/0403/18, KEGA 018TUKE-4/2016.

\section{References}

1. S. Best, M. Riseborough, Automated people movers and automated transit systems (ASCE, New York, 2018)

2. P. J. Murtagh, R. S. Vecchio, D. J. McFadden, Automated people movers and automated transit systems (ASCE, New York, 2018) 
3. P. Ravotti, B. Adams, Automated people movers and automated transit systems (ASCE, New York, 2018)

4. J. Schuette, S. Scholz, C. Crawford, 75th Vehicular technology conference (IEEE, Québec City, 2012)

5. I. Dockalikova, K. Kashi, 8th. Int. D. Stat. E 14, 7 (2014)

6. I. Kubasakova, R. Kampf, O. Stopka, Kom. Ek. 8, 11 (2014)

7. L. Bartuska, O. Stopka, M. Chovancova, J. Lizbetin, 20th. Int. S. Conf. Trans. M. 20, 5 (2016)

8. https://www.coreconagvs.com/images/products/P330.jpg

9. https://www.coreconagvs.com/industries_served/images/Special-PeopleMover.jpg

10. G. Ulrich, Automated Guided Vehicle Systems (Springer, Berlin, 2015)

11. R. Kampf, S. Lorincova, M. Hitka, O. Stopka. Sustainability, 9. DOI: $10.3390 / \mathrm{su} 9091561$ (2017) 